\title{
Ethnic background and risk of SARS-CoV-2 infection among the healthcare workforces: Results of a large occupational cohort study in rural United Kingdom.
}

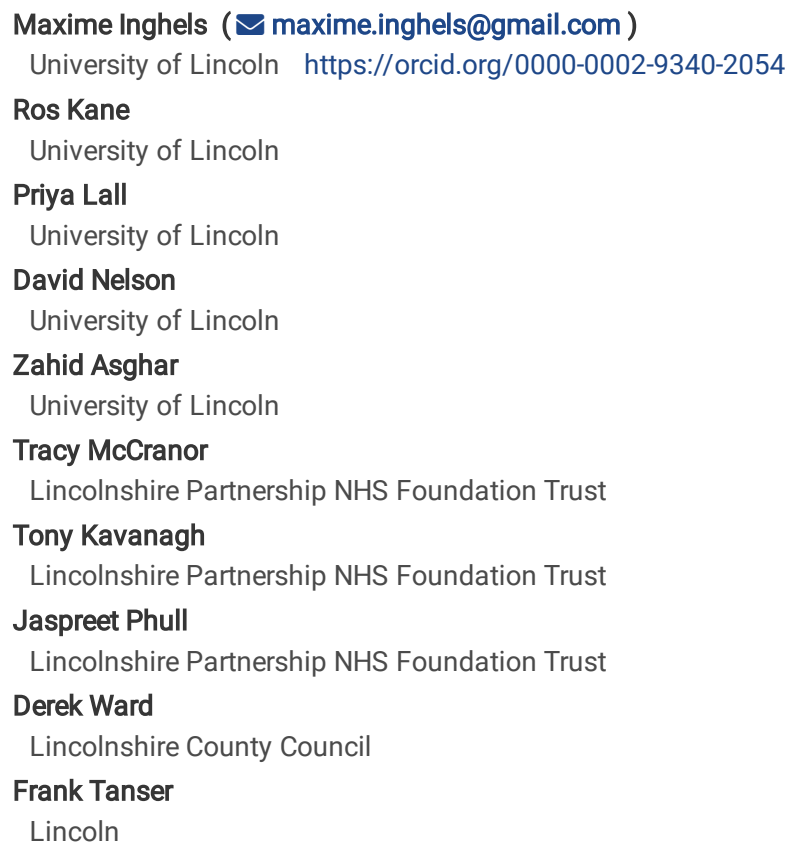




\section{Abstract}

Black and South Asian healthcare workers have been found at higher risk of SARS-CoV-2 infection in the United Kingdom. However, many studies do not consider all potential confounders (e.g., professional exposure, living environment) and those conducted during the second wave of the COVID-19 outbreak remain scarce. Using 1-year-folow-up data from a cohort of 13,366 healthcare workers employed in 119 facilities in Lincolnshire, we aimed to quantify the risk of SARS-CoV-2 infection among ethnic minority healthcare staff and to elucidate pathways of infection. Overall, 1258 individuals (9.4\%) recorded a positive SARS-CoV-2 test during the observation period, incidence per person-year was 5.2\% [Cluster adjusted 95\% Cl: 3.6-7.6\%] during the first COVID-19 wave (JanAug 2020) and 17.2\% [13.5-22.0\%] during the second wave (Sep 2020-Feb 2021). Compared to Whites, Black and South Asian employees were at higher risk of SARS-CoV-2 infection during both the first wave (Clustered adjusted Hazard Ratio, 1.58 [0.91-2.75] and 1.69 [1.07-2.66] respectively) and the second wave (HR 2.09 [1.57-2.76] and 1.46 [1.24-1.71]). Higher risk of SARS-CoV-2 infection significantly persisted even after controlling on age, gender, pay grade, residence environment, type of work and time exposure at work. Higher adjusted risk of SARS-CoV-2 infection were also found among lower-paid health professionals. Black and South Asian health workers continue to be more exposed to SARS-CoV-2 infection compared to their white counterparts. Urgent interventions are required to reduce SARS-CoV-2 exposure with these ethnic groups.

\section{Introduction}

On March 11, 2020, the World Health Organization (WHO) declared the novel Severe acute respiratory syndrome coronavirus 2 (SARS-CoV-2) outbreak a global pandemic ${ }^{1}$. Coronavirus disease (COVID-19), caused by SARS-CoV-2, has affected more than 178 million individuals worldwide, including 4.6 millions of cases in the UK ${ }^{2}$. Because they are at the frontline of the outbreak, healthcare workers are particularly exposed to SARS-CoV-2 ${ }^{3,4}$. In the UK, at the early stages of the outbreak, a study conducted among 200 high-risk frontline medical staff showed that $25 \%$ were already positive at study entry and $20 \%$ seroconverted during the following two weeks ${ }^{5}$. Despite the supply of personal protective equipment, the adoption of social distancing and shielding isolation measures, health services staff continue to be more affected by COVID-19 compared to the general population ${ }^{4,6}$.

All healthcare staff do not carry the same risk of infection. In the UK, studies indicated that professionals in face-to-face contact with patients (e.g. porters, healthcare assistants and nurses) are more likely to be infected by SARS-CoV-2 than those who are not ${ }^{7,8}$. Furthermore, UK healthcare professionals from Black (i.e. African or Caribbean) and South Asian (i.e. Pakistanis, Indians, Bangladeshis) backgrounds have displayed a higher risk of SARS-CoV-2 infection ${ }^{4,7-16}$. However, many studies investigating risk factors for SARS-CoV-2 infection have limitations, such as cross-sectional designs unable to demonstrate causality ${ }^{8,13-16}$, a limited number of health facilities included questioning study representativeness ${ }^{11,13,14}$, unadjusted results or omission of important confounding variables (e.g., occupational exposure, residential environment) ${ }^{9,12,14,15}$.

A key outstanding question in this area is "to what extent are the observed differences in ethnicity risk explained by differences in job profile and other socioeconomic and environmental conditions?". In the UK, low economic status has been shown to be associated with higher COVID-19 prevalence in the general population 17,18 , but such association remains unclear among health professionals ${ }^{8,10,12,13}$. Furthermore, in these studies, the socioeconomic factors that are considered are mainly area based and other individual-based variables such as professional grade or salary are rarely considered. In addition, SARS-CoV-2 infection risks in rural environments remained poorly described in $\mathrm{UK}^{19}$.

In order to quantify changing patterns of infection risk, one needs to go beyond the first wave of the pandemic. However, to date, there are only two peer reviewed publications examining the impact of the second wave of the outbreak on healthcare professionals in the UK. The first focused on dental care professionals; while the second was a cross-sectional study in a single hospital setting ${ }^{9,11}$. Understanding the evolution of SARS-CoV-2 infection risk factors across the different waves of the pandemic is important to observe if the generalisation and adoption of mitigation measures (e.g. provision of personal protective equipment, vaccination roll-out) have reduced sociodemographic and economic risk inequalities between healthcare staff. The aim of this study was to quantify risk of COVID-19 infection among ethnic minority healthcare workers and elucidate pathway of infection by considering underlying differences in demographics, socioeconomic status, residential environment and occupational exposure.

\section{Results}

Overall, we collected data from 13,880 healthcare professionals. Among them, 56 were documented as working in more than one medical facility and 44 were not based in any facilities and were thus excluded from the analysis. Additionally, we removed 58 students as their staff group were not mentioned. An additional 356 individuals with missing values have been removed leading to 13,366 individuals included in our final sample. The selection process leads to the exclusion of 4 of the initial 123 health facilities present in the datasets. The mean average follow-up by individual was 356 days; only $24 \%$ of the individuals included were followed less than 407 days (the maximum follow-up).

The cohort comprised mainly women (79.8\%, Table 1$)$. White individuals represented $87.9 \%$ of the cohort while South Asian and Black employees represented $5.3 \%$ and $1.9 \%$ respectively. Registered nurses and midwives were the most frequent staff group (27.8\%), followed by additional clinical services staff ( $21.9 \%)$ and administrative and clerical staff (21.9\%). Employees included at the first COVID-19 wave have similar demographic characteristics to those included in the second wave (Supplementary material, Table S1)

There were 1,258 individuals (9.4\%) with a documented SARS-CoV-2 positive test (Table 1). Among them, 28 ( $2.2 \%)$ had a second positive test more than 90 days after the first one, only the first SARS-CoV-2 test was considered for them. Facilities with the higher number of employees reported the majority of the positive SARS-CoV-2 tests (Figure 1). Incidence of SARS-CoV-2 positive test during the observation period was highly heterogenous between health facilities (Supplementary materials, Figure S1). For example, one county hospital had $26.2 \%$ of their staff who were tested positive for SARS-CoV-2 compared to $4.6 \%$ in another one. 
Population characteristics description and SARS-CoV-2 prevalence during the study period $(n=13,366)$.

\begin{tabular}{|c|c|c|c|c|c|}
\hline & \multicolumn{2}{|c|}{ Headcount } & \multicolumn{2}{|c|}{ Tested positive for SARS-CoV-2 } & \multirow[t]{2}{*}{ p-value ${ }^{1}$} \\
\hline & $\mathbf{N}$ & $\%$ & $\mathrm{n}$ & $\%(n / N)$ & \\
\hline Sex & & & & & $<0.001$ \\
\hline Female & 10667 & 79.8 & 1062 & 10.0 & \\
\hline Male & 2699 & 20.2 & 196 & 7.3 & \\
\hline Age & & & & & 0.002 \\
\hline 30 and below & 2619 & 19.6 & 259 & 9.9 & \\
\hline $31-40$ & 2976 & 22.3 & 290 & 9.7 & \\
\hline $41-50$ & 3000 & 22.4 & 307 & 10.2 & \\
\hline $51-60$ & 3462 & 25.9 & 317 & 9.2 & \\
\hline 61 and over & 1309 & 9.8 & 85 & 6.5 & \\
\hline Ethnic group & & & & & 0.009 \\
\hline White - British & 11248 & 84.2 & 1035 & 9.2 & \\
\hline Other White minorities & 499 & 3.7 & 48 & 9.6 & \\
\hline Southern Asian ${ }^{2}$ & 707 & 5.3 & 83 & 11.7 & \\
\hline Other Asian background & 249 & 1.9 & 23 & 9.2 & \\
\hline Black $^{3}$ & 250 & 1.9 & 36 & 14.4 & \\
\hline Mixed and other & 413 & 3.1 & 33 & 8.0 & \\
\hline Staff group & & & & & $<0.001$ \\
\hline Additional Clinical Services & 2926 & 21.9 & 399 & 13.6 & \\
\hline Administrative and Clerical & 2925 & 21.9 & 113 & 3.9 & \\
\hline Allied Health Professionals & 784 & 5.9 & 71 & 9.1 & \\
\hline Estates and Ancillary & 1111 & 8.3 & 87 & 7.8 & \\
\hline Medical and Dental & 1250 & 9.4 & 96 & 7.7 & \\
\hline Nursing and Midwifery Registered & 3719 & 27.8 & 458 & 12.3 & \\
\hline Scientists, Prof Scientific and Technic & 654 & 4.9 & 34 & 5.2 & \\
\hline Pay grade & & & & & 0.072 \\
\hline NHS Body Band 1, 2 and 3 & 4945 & 37.0 & 509 & 10.3 & \\
\hline NHS Body Band 4 and 5 & 3310 & 24.8 & 355 & 10.7 & \\
\hline NHS Body Band 6 and 7 & 3097 & 23.2 & 263 & 8.5 & \\
\hline NHS Body Band 8,9 and others ${ }^{4}$ & 2014 & 15.1 & 131 & 6.5 & \\
\hline Contract type & & & & & 0.179 \\
\hline Part-time & 5809 & 43.5 & 523 & 9.0 & \\
\hline Full-time & 7557 & 56.5 & 735 & 9.7 & \\
\hline Index of multiple deprivation & & & & & 0.119 \\
\hline
\end{tabular}

\begin{tabular}{|l|}
\hline $\begin{array}{l}1 \text { two-sided global p-values (i.e. both rows and columns comparison for each characteristics) were computed using cluster-adjusted chi-square test of } \\
\text { independence }\end{array}$ \\
\hline 2 Include Pakistanis, Indians, Bangladeshis \\
\hline${ }^{3}$ Include Black African and Caribbean \\
\hline${ }^{4}$ Other pay grades encompass mainly specialty registrar and consultant \\
\hline${ }^{5}$ Measured during the study period \\
\hline
\end{tabular}




\begin{tabular}{|c|c|c|c|c|c|}
\hline & Headcount & & Tested pos & S-CoV-2 & $p$-value ${ }^{1}$ \\
\hline lower decile (1-4) & 4396 & 32.9 & 432 & 9.8 & \\
\hline middle decile (5-7) & 4433 & 33.2 & 448 & 10.1 & \\
\hline upper decile (8-10) & 4537 & 33.9 & 378 & 8.3 & \\
\hline Urban-rural classification & & & & & 0.822 \\
\hline Urban & 8062 & 60.3 & 763 & 9.5 & \\
\hline Rural & 5304 & 39.7 & 495 & 9.3 & \\
\hline Number of non-COVID diseases & & & & & $<0.001$ \\
\hline 0 & 5561 & 41.6 & 404 & 7.3 & \\
\hline 1 & 3451 & 25.8 & 309 & 9.0 & \\
\hline 2 & 2177 & 16.3 & 244 & 11.2 & \\
\hline $3+$ & 2177 & 16.3 & 301 & 13.8 & \\
\hline Number of isolation days ${ }^{5}$ & & & & & $<0.001$ \\
\hline 0 & 8671 & 64.9 & 712 & 8.2 & \\
\hline$[1-14]$ & 3225 & 24.1 & 455 & 14.1 & \\
\hline$>14$ & 1470 & 11.0 & 91 & 6.2 & \\
\hline Other leaving absence days ${ }^{5}$ & $N A=1821$ & & $N A=262$ & & 0.001 \\
\hline 0 & 7789 & 67.5 & 736 & 9.4 & \\
\hline$[1-7]$ & 2784 & 24.1 & 203 & 7.3 & \\
\hline$>7$ & 972 & 8.4 & 57 & 5.9 & \\
\hline \multicolumn{6}{|c|}{$\begin{array}{l}1 \text { two-sided global p-values (i.e. both rows and columns comparison for each characteristics) were computed using cluster-adjusted chi-square test of } \\
\text { independence }\end{array}$} \\
\hline \multicolumn{6}{|c|}{${ }^{2}$ Include Pakistanis, Indians, Bangladeshis } \\
\hline \multicolumn{6}{|l|}{${ }^{3}$ Include Black African and Caribbean } \\
\hline \multicolumn{6}{|c|}{${ }^{4}$ Other pay grades encompass mainly specialty registrar and consultant } \\
\hline${ }^{5}$ Measured during the study period & & & & & \\
\hline
\end{tabular}

The overall SARS-CoV-2 infection incidence per person-year was 10.0\% [cluster adjusted $95 \%$ confidence interval (CI): $8.8-11.4 \%$ ]. During the first wave, the incidence was 5.2\% [3.6-7.6\%], whilst it was 17.2\% [13.5-22.0\%] during the second wave. Incidence was higher for Black employees (18.6\% [13.7-25.3\%]) and the South Asian ones (14.7\% [11.9-18.1\%]) compared to Whites (9.5\% [8.3-11.0\%]). Higher SARS-CoV-2 incidence was also found among additional clinical service staff (14.8\% [11.7-18.7\%]) and nurses (12.4\% [11.0-14.1\%]).

Using Kaplan-Meyer curves, we found that Black employees had significantly higher probability to test positive for SARS-CoV-2 infection compared to Whites at 6 months of follow-up $(7.1 \%$ [2.6-11.4\%] vs 3.4\% [2.3-4.5\%], p=0.001) and at the end of the observation period $(20.9 \%$ [14.5-26.8\%] vs $10.5 \%$ [9.0-12.0\%], p < 0.001) (Figure 2.A.). Similar results were found for South Asian employees (6.8\% [3.9-9.5\%] vs 3.4\% [2.3-4.5\%], p=0.001, at 6 months and, $15.8 \%$ [13.5$18.0 \%$ ] vs $10.5 \%$ [9.0-12.0\%], p $<0.001$, at the end of the observation period). Probability of SARS-CoV-2 infection was significantly different by staff group with additional clinical services staff the group having the higher probabilities of infection at 6 month and at the end of the observation period (respectively, $5.6 \%$ [4.0-7.2\%] and 15.9\% [12.1-19.5\%]) (Figure 2.B.). Women and employees with lower NHS pay grades were also found with a significantly higher probability for SARS-CoV-2 infection (Figure 2.C. and 2.D.).

Compared to Whites, Black and South Asian employees were at higher risk of SARS-CoV-2 positive test during both the first wave (Clustered adjusted Hazard Ratio, HR 1.58 [0.91-2.75] and 1.69 [1.07-2.66] respectively) and the second wave (HR 2.09 [1.57-2.76] and 1.46 [1.24-1.71]) (Figure 3, associated tables can be found in supplement material, Tables S1 and S2). These higher risks of SARS-CoV-2 infection among Black and South Asian employees were still significant after adjusting on age, sex, NHS pay grad, staff group, contract type, isolation or leave periods, number of non-COVID-19 related diseases, IMD and urban-rural classification of the residence.

Whilst age was not found to be significantly associated with a higher risk of SARS-CoV-2 infection during the first wave of the outbreak, those who were 60 years old and over were at lesser risk of positive test during the second wave (vs less than 30 years old, $\left.\mathrm{HR} 0.52 \mathrm{Cl}_{95 \%}[0.43-0.65]\right)(\mathrm{Table} \mathrm{S} 2)$.

Women (aHR 1.27 [1.06-1.52]) and employees with the lowest paygrade (vs highest, aHR 3.13 [2.08- 4.55]) were at higher risk of SARS-CoV-2 infection. Both rural-urban classification and IMD were not found to be significantly associated with SARS-CoV-2 infection.

Page $4 / 11$ 
Experiencing a number of isolation days for two weeks or less was significantly associated with a higher risk of positive SARS-CoV-2 test (vs none, aHR 2.16 $\mathrm{Cl}_{95 \%}[1.80-2.60]$ ), but not when that number exceeded two weeks (vs none, aHR $0.97 \mathrm{Cl}_{95 \%}[0.82-1.15]$ ). Those experiencing 2 or more non COVID-19 related diseases were also more at risk of positive SARS-CoV-2 test (vs none, aHR $1.28 \mathrm{Cl}_{95 \%}[1.09-1.51]$ ).

In the complete case analysis, results from the Cox multivariate models were similar to those previously presented except for IMD of the healthcare workers residence which was significantly associated with SARS-CoV-2 infection in the complete case analysis, although the effects remained small (Figure S2).

\section{Discussion}

Our results demonstrated a $110 \%$ excess risk of SARS-CoV-2 infection among Black healthcare workers (relative to their white counterparts) in a large occupational cohort in the predominantly rural English county of Lincolnshire. The corresponding figures for South Asian healthcare workers was more than $60 \%$. These excess risks were not fully explained by demographics, economic situation, occupational exposure, clinical factors or home environment. Whilst SARS-CoV-2 infection incidence increased during the second wave of the pandemic, risk groups remained similar between the first and second COVID-19 outbreak waves. Only professionals of older age were at lower risk of positive SARS-CoV-2 test during the second wave of the outbreak.

One of the major contributions of our study is to show the higher risk of being infected by the SARS-CoV-2 virus among ethnic minorities during both outbreak waves of the pandemic. Our results support previous findings showing higher risk of SARS-CoV-2 infection among Black and South Asian healthcare workers in the UK ${ }^{4,7-16}$. Yet, our research is one of the first to have considered more than 1 year of follow-up and found no changes in SARS-CoV-2 exposure by ethnic group between waves. While Black and Asian communities were considered to be at higher risk for SARS-CoV-2 infections during the first wave of the pandemic, their persistent higher risk during the second wave, suggests that interventions aimed at protecting them were either absent or either failed in reducing their occupational risk ${ }^{20,21}$. Considering the fact that these communities tend to have worse clinical outcomes when tested positive for COVID-1917,22, tailored interventions, as well as, improved access to vaccination are urgently needed to protect ethnic minority essential workers ${ }^{21,23}$.

A second contribution of this paper is to show persistent infection risk among Black and South Asian communities even after adjusting on demographics, economic situation, occupational exposure, clinical factors or home environment, that may suggest potential biological susceptibility of these populations. Several studies has identified several genes that could explained differences in SARS-CoV-2 infection susceptibility between ethnic groups ${ }^{24,25}$. However, the effect size of the genetic contrition to the overall number of new infections remains undocumented.

Additional clinical services staff were at higher risk of SARS-CoV-2 infection. This staff group includes a range of professionals that are in close contact with patients (e.g. healthcare assistants, support workers). The fact that they are more exposed compared to other professionals in close contact with patients (e.g. nurses) could suggest that they do not benefit the same level of protection (e.g. access to personal protection equipment) or awareness on mitigation measures (e.g. physical distance). Yet, the duties of additional clinical services staff (e.g. personal care) can be challenging with the adoption and maintaining of mitigation measures. Additionally, this staff group may not benefit from the same level of consideration when it comes to the national guidelines compared to other professionals like physicians or nurses. Therefore, future guidance should consider more explicitly the diverse range of professions that make up and contribute to the delivery of front-line care.

Our findings reinforce the results from other studies showing the limited effect of IMD among healthcare professionals ${ }^{10,13}$. IMD is an area-level indicator rather than individual level socioeconomic-position which may not be representative of every individual, especially those currently working and who may be less likely to be affected by the environment in which they live. However, after adjusting on other individual characteristics and staff group, we did find that having a lower salary was associated with the risk of being tested positive for SARS-CoV-2. This highlights unacceptable inequalities in a sector where everyone belonging to the same staff group should benefit from the same level of protective measures.

Compared to the first wave, older people were at lesser risk during the second wave of the outbreak. This could suggest that measures adopted during the first wave to protect older and vulnerable people were potentially effective during the second wave of the outbreak. It could also indicate better access to a COVID19 vaccination, as vaccines were made available for frontline healthcare professionals in December $2020^{26}$.

Another strength of our analysis is to have considered exposure time at work. As expected, those spending more time at work and those who did not take any leave days were those more likely to be tested positive for SARS-CoV-2, which suggests that these professionals were mainly infected at work and not outside of their workplace. However, the fact that those in leave for infection precaution reason for two weeks or less were more at risk of getting a positive test may indicate those not yet tested positive but who have been in close contact which someone infected and asked to self-isolate. Therefore, it is reassuring to know that short isolation for infection precaution can potentially reduce the number of SARS-CoV-2 infections within the workplace.

Our study has several limitations. Firstly, we were not able to access data on the number of tests performed by individuals and specifically, the negative ones. Thus, it was not possible to control our results on the number of tests done by individuals. However, ethnic differences in testing are shown to be small during the first two waves of the pandemic ${ }^{22}$. Therefore, the higher risk of a positive SARS-CoV-2 test among ethnic minorities is unlikely to be a result of a higher number of tests performed. Another limitation of our study is the fact that we partially take into account the clinical status of a professional, especially data on underlying conditions. The reason for sick leave was not available for more of our population, thus we were not able to consider specific conditions (e.g. diabetes, cardiovascular disease), although these chronic conditions are associated with a higher risk of COVID-19 related hospitalisation and mortality ${ }^{27-29}$, but not to a higher risk of infection ${ }^{9,16}$. Other variables not available in the data that could have been considered to our model are the access and the use of personal protective equipment ${ }^{4,10}$. Because of the limited sample of an individual experiencing a re-infection, we did not consider those who experienced a subsequent positive SARS-CoV-2 test more than 3 months after the first one. 
Nevertheless, our survey is one of the first to document SARS-CoV-2 exposure across one year of follow-up among a large population of healthcare professionals at a regional level. The fact that we included the three NHS trusts operating within the region has facilitated a comprehensive sample. Yet it is possible that private sector structures as well as several GP surgeries may not have been included in our study. Because most of these structures were doing online consultation or referring people to the hospital in case of severe symptoms of COVID-19, professionals working in these structures could have been less exposed to SARS-CoV-2 infections than those working in a hospital setting.

Our analysis only focused on the health workforce in the Lincolnshire region which is a predominantly rural county and may therefore not be generalisable to all of the UK. However, we included a high number of various structures (e.g., GP practices, hospital, health centres) providing different type of health services (metal health, acute care and community care) which allowed us to take into account the richness of healthcare sector where other studies mainly focused on teaching or university hospitals $\mathbf{s}^{7,11,14,30,31}$.

Although we did not collect data on SARS-CoV-2 vaccination coverage in our study, evidence indicates low vaccine coverage among healthcare workers especially with Black and south Asian groups ${ }^{32}$. Vaccine hesitancy has been shown to be higher in ethnic minority groups as well as in the most deprived areas $^{33,34}$. This disparity in vaccination uptake could lead to the increasing risk of SARS-CoV-2 infection in these communities during future waves of the outbreak. Reducing access inequalities to protective intervention and measures is urgently needed.

\section{Methods}

Design and Settings

An occupational cohort study has been conducted among all employees (both clinical and non-clinical) working at 123 facilities in Lincolnshire, a predominantly rural region in UK. Staff are employed at one of three hospital trusts namely: Lincolnshire Partnership NHS Foundation Trust, Lincolnshire Community Health Services and United Lincolnshire Hospitals NHS Trusts. These facilities include 11 hospitals, 44 GP medical practices or health centres, 47 other medical facilities (e.g. hospice, centre for mentally ill patient) and 21 non-medical health facilities. Majority of staff are hospital-based (72.3\%).

Population included

From the 1st January 2020 to the 10th February 2020, all employees working in the three trusts were retrospectively followed. Following UK National Health Service (NHS) recommendations, before November 2020, NHS staff were tested using PCR-based SARS-CoV-2 tests in case of COVID-19 related symptoms or contact with COVID-19 cases $^{35}$. Since November 2020, the NHS provided lateral Flow antigen test kits and recommended that staff performed regular SARSCoV-2 tests (at least twice a week) and to perform a PCR confirmation test in case of positive result. Positive test results were recorded by the human resources department of each Trust. Students working in these facilities were excluded because their staff group assignment was not reported in the datasets provided. Healthcare staff documented as working in different facilities or those not assigned to a specific facility during the same period were also excluded from the study. Individuals belonging to more than one staff group during a given period were assigned to the staff group where the individual spent most of their time.

Data collected

To fully understand pathways of SARS-CoV-2 infection, it was important to collect a large suite of variables related to demographics (i.e., age, sex, ethnic group), economic situation (i.e., NHS pay grad), occupational exposure (i.e., staff group, number of hours worked each weekend, self or house isolation periods and leaving absence periods), clinical factors (i.e., number of non-COVID-19 related diseases occurring during the observation period) and home environment (i.e., UK Index of multiple deprivation-IMD, and UK urban-rural classification). Ethnic groups were categorised as Whites-British, other White minorities (e.g., Europeans), Southern Asians (i.e., Pakistanis, Indians and Bangladeshis), other Asian minorities, Blacks (including Black African and Caribbean) and Mixed groups or others. NHS pay grad vary from NHS body band 1 to 9 with band 1 indicating lower salaries (starting at around 24,830 US dollars per year) while the highest band indicate a higher salary (starting at around 125,483 US dollar per year). The rural-urban classification from 2010 and the IMD from 2019 were linked for each individual by merging the lower layer super output area associated to their home postcode with UK census data. Leaving absences (excluding infection precaution or isolation) and self or house isolation periods (including COVID-19 shielding or infection precaution reasons) were measured through the start and end date of these periods.

The first wave (i.e. wave 1) was defined as the period from the 1st of January 2020 -31st August 2020 and the second wave, the period between the 1 st September 2020 - 10th February $2021^{22}$.

\section{Statistical analysis}

Firstly, descriptive statistics were used to provide an overview of the data collected in the included sample. Bivariate analysis of SARS-CoV-2 positive test prevalence by the collected variables was conducted. SARS-CoV-2 positive test incidence rates per person-year were documented and cluster-adjusted on the place of work.

Survival analysis was conducted to measure the hazard risk ratio of positive SARS-CoV-2 test occurrence. For each individual, observation period was left truncated by the start date of the study observation (i.e., 1st January 2020) or the start date of employment contract if that date occurred after the beginning of the study observation. The observation period was also right truncated by the study observation period end date (i.e., 10th February 2020), the end of the contract date or the date of occurrence of the first positive SARS-CoV-2 test. 
Bivariate analysis between a SARS-CoV-2 positive test and other variables of interest was conducted using the log-rank test on Kaplan-Meier estimator ${ }^{36}$. Then, we ran bivariate and multivariate cox regression models depending on the period of observation: all the study period, wave 1 and wave $2^{37}$. All initial variables of interest were included in the multivariate model. Because data on leaving absence (other than COVID-19 related) was not available for a trust representing $13.7 \%$ of our sample, we assumed this value to be zero in the Cox models to avoid sample attrition. Complete case analysis for bivariate and multivariate models (i.e., exclusion of the individuals with missing values for the number of leaving absence days) has been conducted for sensibility analysis; related results are presented in supplementary materials.

To consider the clustering formation feature of SARS-CoV-2 infection, we added a cluster effect on the place of work in the Cox models. Analysis were conducted using the package "survival" from the software R version 4.0.2. ${ }^{38,39}$.

Ethical consideration

Data sharing agreements were signed with each trust who provided their data. An anonymized ID have been provided to each individual and name of work locations have been anonymised to avoid potential confidentiality breach. The study received a favourable ethical opinion from the Human Ethics Committee at the University of Lincoln on the 28 May 2020 (REC Ref: 2020-3511).

Patient and Public Involvement

Whilst no patients or members of the public were directly involved in the study, the research was overseen, and quality assessed by senior healthcare staff (clinical, non-clinical) at each participating NHS trust who were instrumental with supporting the research from its early planning stages right through to devising the dissemination and impact strategy. We plan to disseminate the results with the participating NHS trusts, as well as, with the wider healthcare workforce, both regionally and nationally. Finally, the findings will also be reported to the general public via a range of non-academic outputs (e.g., social media, press releases, accessible summaries).

\section{Data availability}

The study data are not publicly available. However, access to the anonymised dataset can be obtained upon approval from participating NHS Trusts. The corresponding author can liaise with the point of contact in each Trust upon reasonable request.

\section{Code availability}

Code related to the analysis can be provide upon request to the corresponding author.

\section{Declarations}

Competing interest statement: All authors have completed the Unified Competing Interest form (available on request from the corresponding author) and declare: no support from any organisation for the submitted work; no financial relationships with any organisations that might have an interest in the submitted work in the previous three years, no other relationships or activities that could appear to have influenced the submitted work.

Author contributions: RK, JP and FT conceived the study. MI, RK, JP and FT contributed to the study design and protocol development. MI, RK, TMC and TK obtained the data. MI analysed the data. MI, RK, PL, DN, ZA and FT contributed to the interpretation of the results. MI and RK had full access to all the data and take responsibility for the integrity of the data as well as the accuracy of the data analysis. MI, RK, PL and DN wrote the first draft of the manuscript. All authors provided substantive feedback on the manuscript and have read and approved the final version. MI is the guarantor. The corresponding author attests that all listed authors meet authorship criteria and that no others meeting the criteria have been omitted.

Transparency declaration: the lead author affirms that the manuscript is an honest, accurate, and transparent account of the study being reported; that no important aspects of the study have been omitted; and that any discrepancies from the study as planned (and, if relevant, registered) have been explained.

Ethical approval: The study only used routinely collected data by the participating NHS trusts and data sharing agreements were signed between the University of Lincoln and each trust. The study received a favourable ethical opinion from the Human Ethics Committee at the University of Lincoln on the 28 May 2020 (REC Ref: 2020-3511).

Funding statement: The study received no direct funding.

Acknowledgement: We would like to thank all participating NHS Trusts and implementing partners who supported the dissemination of the survey.

\section{References}

1. WHO Director-General's opening remarks at the media briefing on COVID-19-11 March 2020. https://www.who.int/directorgeneral/speeches/detail/who-director-general-s-opening-remarks-at-the-media-briefing-on-covid-19--11-march-2020.

2. WHO Coronavirus (COVID-19) Dashboard. https://covid19.who.int.

3. Hunter, E. et al. First experience of COVID-19 screening of health-care workers in England. The Lancet 395, e77-e78 (2020). 
4. Nguyen, L. H. et al. Risk of COVID-19 among front-line health-care workers and the general community: a prospective cohort study. Lancet Public Health 5, e475-e483 (2020).

5. Houlihan, C. F. et al. Pandemic peak SARS-CoV-2 infection and seroconversion rates in London frontline health-care workers. The Lancet 396, e6-e7 (2020).

6. Office for National Statistics. Coronavirus (COVID-19) related deaths by occupation, England and Wales. Office for National Statistics website https://www.ons.gov.uk/peoplepopulationandcommunity/healthandsocialcare/causesofdeath/bulletins/coronaviruscovid19relateddeathsbyoccupationenglar (2021).

7. Eyre, D. W. et al. Differential occupational risks to healthcare workers from SARS-CoV-2 observed during a prospective observational study. eLife 9, e60675 (2020).

8. Martin, C. A. et al. Demographic and occupational determinants of anti-SARS-CoV-2 IgG seropositivity in hospital staff. J. Public Health Oxf. Engl. fdaa199 (2020) doi:10.1093/pubmed/fdaa199.

9. Shields, A. M. et al. COVID-19: Seroprevalence and Vaccine Responses in UK Dental Care Professionals. J. Dent. Res. 00220345211020270 (2021) doi:10.1177/00220345211020270.

10. Valdes, A. M. et al. Longitudinal assessment of symptoms and risk of SARS-CoV-2 infection in healthcare workers across 5 hospitals to understand ethnic differences in infection risk. EClinicalMedicine 34, 100835 (2021).

11. Ken-Dror, G. et al. SARS-CoV-2 antibody seroprevalence in NHS healthcare workers in a large double-sited UK hospital. Clin. Med. 21, e290-e294 (2021).

12. Hanrath, A. T. et al. SARS-CoV-2 Testing of 11,884 Healthcare Workers at an Acute NHS Hospital Trust in England: A Retrospective Analysis. Front. Med. $8,636160(2021)$.

13. Shields, A. et al. SARS-CoV-2 seroprevalence and asymptomatic viral carriage in healthcare workers: a cross-sectional study. Thorax 75, 1089-1094 (2020).

14. Shorten, R. J. et al. Seroprevalence of SARS-CoV-2 infection in healthcare workers in a large teaching hospital in the North West of England: a period prevalence survey. BMJ Open 11, e045384 (2021).

15. Patel, M., Nair, M., Pirozzoli, E., Cienfuegos, M. C. \& Aitken, E. Prevalence and socio-demographic factors of SARS-CoV-2 antibody in multi-ethnic healthcare workers. Clin. Med. 21, e5-e8 (2021).

16. Kua, J. et al. healthcareCOVID: a national cross-sectional observational study identifying risk factors for developing suspected or confirmed COVID-19 in UK healthcare workers. PeerJ 9, e10891 (2021).

17. Chaudhuri, K., Chakrabarti, A., Lima, J. M., Chandan, J. S. \& Bandyopadhyay, S. The interaction of ethnicity and deprivation on COVID-19 mortality risk: a retrospective ecological study. Sci. Rep. 11, 11555 (2021).

18. Ho, F. K. et al. Modifiable and non-modifiable risk factors for COVID-19, and comparison to risk factors for influenza and pneumonia: results from a UK Biobank prospective cohort study. BMJ Open 10, e040402 (2020).

19. Asghar, Z. B., Ward, D., Huntly, P., Inghels, M. \& Tanser, F. COVID-19 (SARS-CoV-2) Outbreaks in Rural United Kingdom: Patterns of Spread and Empirical Evidence for the 'Rule of Six'. https://papers.ssrn.com/abstract=3807267 (2021) doi:10.2139/ssrn.3807267.

20. Public Health England. Disparities in the risk and outcomes of COVID-19. 92

https://assets.publishing.service.gov.uk/government/uploads/system/uploads/attachment_data/file/908434/Disparities_in_the_risk_and_outcomes_of_COVII

21. lacobucci, G. Covid-19: PHE review has failed ethnic minorities, leaders tell BMJ. BMJ 369, m2264 (2020).

22. Mathur, R. et al. Ethnic differences in SARS-CoV-2 infection and COVID-19-related hospitalisation, intensive care unit admission, and death in 17 million adults in England: an observational cohort study using the OpenSAFELY platform. The Lancet 397, 1711-1724 (2021).

23. Race Disparity Unit, Cabinet Office. Third quarterly report on progress to address COVID-19 health inequalities. GOV.UK https://www.gov.uk/government/publications/third-quarterly-report-on-progress-to-address-covid-19-health-inequalities/third-quarterly-report-on-progress-toaddress-covid-19-health-inequalities (2021).

24. Yamamoto, N. et al. SARS-CoV-2 infections and COVID-19 mortalities strongly correlate with ACE1 I/D genotype. Gene 758, 144944 (2020).

25. Lu, C., Gam, R., Pandurangan, A. P. \& Gough, J. Genetic risk factors for death with SARS-CoV-2 from the UK Biobank.

http://medrxiv.org/lookup/doi/10.1101/2020.07.01.20144592 (2020) doi:10.1101/2020.07.01.20144592.

26. Department of Health and Social Care [UK]. Joint Committee on Vaccination and Immunisation: advice on priority groups for COVID-19 vaccination, 30 December 2020. GOV.UK https://www.gov.uk/government/publications/priority-groups-for-coronavirus-covid-19-vaccination-advice-from-the-jcvi-30-december- 
2020/joint-committee-on-vaccination-and-immunisation-advice-on-priority-groups-for-covid-19-vaccination-30-december-2020 (2021).

27. Harrison, E. M. et al. Ethnicity and Outcomes from COVID-19: The ISARIC CCP-UK Prospective Observational Cohort Study of Hospitalised Patients. https://papers.ssrn.com/abstract=3618215 (2020) doi:10.2139/ssrn.3618215.

28. Kabarriti, R. et al. Association of Race and Ethnicity With Comorbidities and Survival Among Patients With COVID-19 at an Urban Medical Center in New York. JAMA Netw. Open 3, e2019795 (2020).

29. Piroth, L. et al. Comparison of the characteristics, morbidity, and mortality of COVID-19 and seasonal influenza: a nationwide, population-based retrospective cohort study. Lancet Respir. Med. 9, 251-259 (2021).

30. Shields, A. M. et al. Serological responses to SARS-CoV-2 following non-hospitalised infection: clinical and ethnodemographic features associated with the magnitude of the antibody response. MedRxiv Prepr. Serv. Health Sci. 2020.11.12.20230763 (2020) doi:10.1101/2020.11.12.20230763.

31. Rivett, L. et al. Screening of healthcare workers for SARS-CoV-2 highlights the role of asymptomatic carriage in COVID-19 transmission. eLife 9 , e58728 (2020).

32. Martin, C. A. et al. Association of demographic and occupational factors with SARS-CoV-2 vaccine uptake in a multi-ethnic UK healthcare workforce: a rapid real-world analysis. medRxiv 2021.02.11.21251548 (2021) doi:10.1101/2021.02.11.21251548.

33. Razai, M. S., Osama, T., McKechnie, D. G. J. \& Majeed, A. Covid-19 vaccine hesitancy among ethnic minority groups. BMJ 372, n513 (2021).

34. Glampson, B. et al. North West London Covid-19 Vaccination Programme: Real-world evidence for Vaccine uptake and effectiveness. medRxiv 2021.04.08.21254580 (2021) doi:10.1101/2021.04.08.21254580.

35. Public Health England. COVID-19: management of staff and exposed patients or residents in health and social care settings. GOV.UK https://www.gov.uk/government/publications/covid-19-management-of-exposed-healthcare-workers-and-patients-in-hospital-settings/covid-19-managementof-exposed-healthcare-workers-and-patients-in-hospital-settings (2021).

36. Kaplan, E. L. \& Meier, P. Nonparametric Estimation from Incomplete Observations. J. Am. Stat. Assoc. 53, 457-481 (1958).

37. Cox, D. R. Regression Models and Life-Tables. J. R. Stat. Soc. Ser. B Methodol. 34, 187-202 (1972).

38. Therneau, T. A package for survival analysis in R. (2021).

39. R Core Team. R: A language and environment for statistical computing. (R Foundation for Statistical Computing, 2021).

\section{Figures}

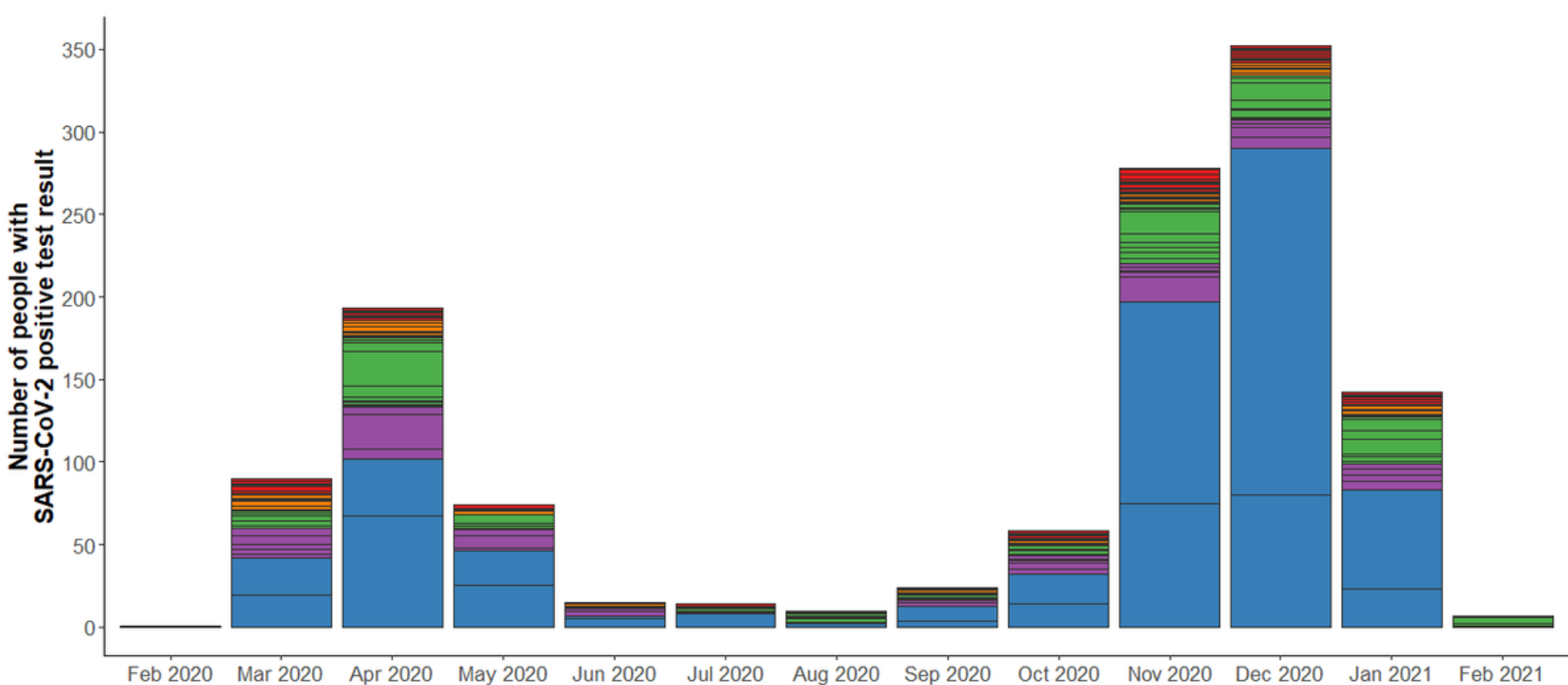

Facilities depending on their number of healthcare staff

$\square$ [0-50] $\square[$ [51-100] $\square$ [101-200] $\square[$ [201-1000] $\square>1000$

Figure 1 
Evolution of the number of positive SARS-CoV-2 test by facility size. Note1: each rectangle represents a facility. The height of the rectangle is depending on the number of documented SARS-CoV-2 positive tests. Note2: Data were obtained through February 10, 2021.
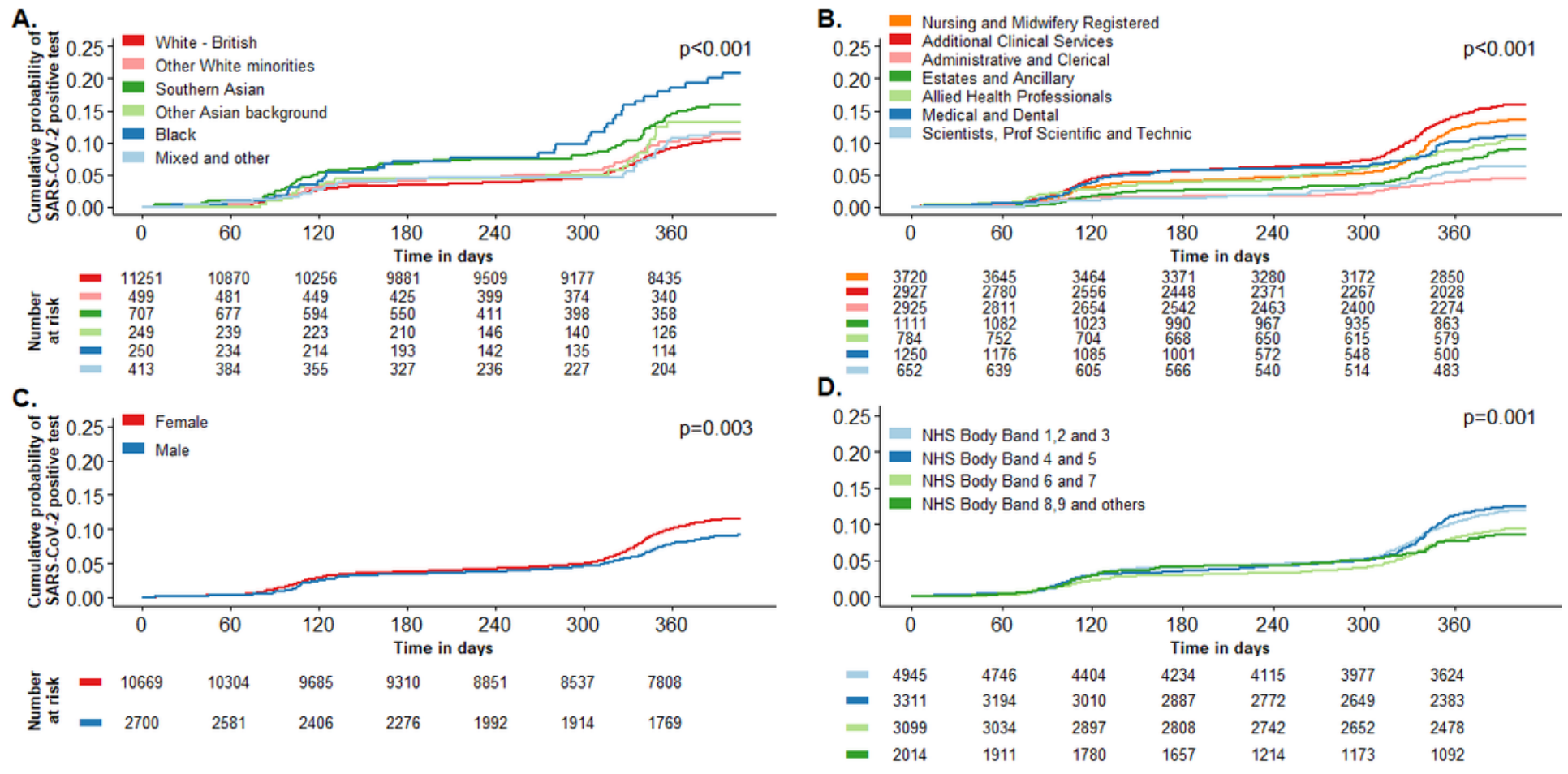

Figure 2

Cumulative event curves, based on Kaplan-Meyer estimates, of COVID-19 positive test among healthcare professional by ethnic background (A), by staff group (B), by sex (C) and NHS salary grade (D). Note1: two-sided p-values were computed using the logrank test. Note2: NHS body band 1 is corresponding to the lower salary grade while NHS body band 9 is corresponding to the higher pay grade

Ethnic Group (Ref. White - British)

Staff Group (Ref. Nursing and Midvifery Registered)
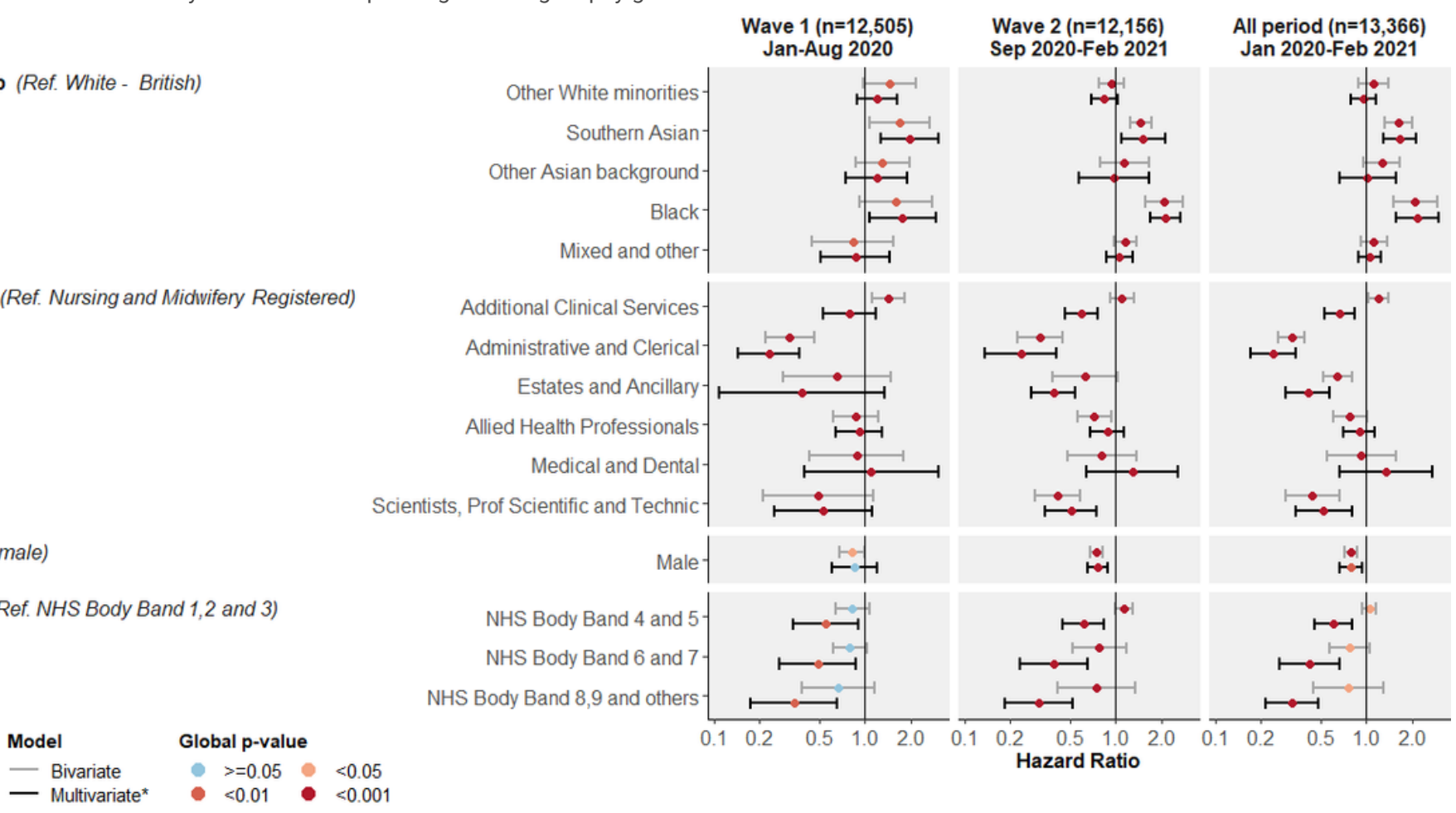

Scientists, Prof Scientific and Technic

Sex (Ref. Female)

Pay Grade (Ref. NHS Body Band 1,2 and 3)

\section{Figure 3}

Hazard ratio of COVID-19 positive test among healthcare professional by time period, bivariate and multivariate cox models. * adjusted on age, contract type, index of multiple deprivation, urban-rural classification, number of Non-COVID diseases, number of isolation days and number of other leaving absence days. The associated result table with all the covariables can be found in Table S2 and Table S3 in supplementary materials Note1: two-sided p-values were 
computed using Wald-test adjusted for clustering. Note2: NHS body band 1 is corresponding to the lower salary grade while NHS body band 9 is corresponding to the higher pay grade Note3: logarithmic scale has been used for the Hazard ratio axis

\section{Supplementary Files}

This is a list of supplementary files associated with this preprint. Click to download.

- STROBEchecklistcohort.doc

- nrreportingsummaryMI.pdf

- Supplementaryfiles.docx 